\title{
Use of Stents for the Maintenance of Hemodialysis Access
}

\author{
Jonathan M. Lorenz, M.D. ${ }^{1}$
}

\section{ABSTRACT}

Maintenance of patent hemodialysis access is critical to prolonging the lives of patients with end-stage renal disease, and metallic stents have become a key tool for this purpose. This article discusses the primary and secondary indications for stent placement in dialysis patients, including angioplasty-induced acute venous rupture, central venous obstruction, early recurring stenosis, elastic stenosis and pseudoaneurysm. The use of stents in patients with synthetic arteriovenous grafts and native arteriovenous fistulae are discussed. With ongoing advances in stent technology as well as brachytherapy, the role of this device in the maintenance of dialysis access will likely increase. An understanding of the indications, precautions, and complications of stent deployment allows the interventional radiologist to become a principle player in the long-term care of dialysis patients.

KEYWORDS: Stent, graft, fistula, dialysis

Objectives: Upon completion of this article, the reader should be able to summarize indications and complications associated with the use of stents to maintain hemodialysis access.

Accreditation: Tufts University School of Medicine (TUSM) is accredited by the Accreditation Council for Continuing Medical Education to provide continuing medical education for physicians.

Credit: TUSM designates this educational activity for a maximum of 1 Category 1 credit toward the AMA Physicians Recognition Award. Each physician should claim only those credits that he/she actually spent in the activity.

Metallic stents play a primary role in maintaining patent hemodialysis access, and as stent technology advances, this role will likely increase. This article will discuss the use of stents in patients with synthetic arteriovenous grafts, native arteriovenous fistulae, and long-term dialysis catheters. Indications such as angioplasty-induced acute venous rupture, central venous obstruction, early recurring stenosis, elastic stenosis and pseudoaneurysm will be discussed. An understanding of the indications, contraindications, and complications of stent deployment will allow the interventional radiol- ogist to retain a principle role in the long-term care of dialysis patients. Maintenance of patent hemodialysis access is critical to prolonging the lives of patients with end-stage renal disease, and metallic stents have become a key tool for preserving the function of a variety of types of permanent dialysis access. Over the last decade, interventional radiologists have described stent placement as a secondary treatment for angioplasty-induced acute venous rupture, early recurring stenosis, and central venous stenosis. In addition, stents have been used as a primary treatment for central venous

Interventional Radiology in Dialysis; Editor in Chief, Peter R. Mueller, M.D.; Guest Editor Brian Funaki, M.D. Seminars in Interventional Radiology, volume 21, number 2, 2004. Address for correspondence and reprint requests: Jonathan M. Lorenz, M.D., Section of Vascular and Interventional Radiology, University of Chicago Hospitals, 5840 S. Maryland Ave, MC2026, Chicago, IL 60637. E-mail: jlorenz@radiology. bsd.uchicago.edu. ${ }^{1}$ Assistant Professor, Section of Vascular and Interventional Radiology, University of Chicago Hospitals, Chicago, Illinois. Copyright (C) 2004 by Thieme Medical Publishers, Inc., 333 Seventh Avenue, New York, NY 10001, USA Tel: +1(212) 584-4662. 07399529,p;2005,22,01,135,140,ftx,en;sir00244. 
occlusion, elastic stenosis, and pseudoaneurysm. With ongoing advances in stent technology as well as brachytherapy, the role of this device in the maintenance of dialysis access will likely increase. This article reviews the applications of stents to the maintenance of synthetic arteriovenous grafts and native arteriovenous fistulae. An understanding of the indications, precautions, and complications of stent deployment allows the interventional radiologist to become a principle player in the long-term care of dialysis patients.

\section{STENT PLACEMENT IN PERIPHERAL VENOUS STENOSES}

\section{Intragraft, Anastomotic and Venous Outflow Stenosis}

All patients with dialysis shunts will eventually face stenosis of the graft, venous anastomosis, or venous outflow. Under-utilizing stents can lead to unnecessary patient morbidity and over-utilizing can lead to additional expense. Studies have shown that the routine use of stents as a first-line treatment of either venous stenosis or recurrence of stenosis after angioplasty is unnecessarily costly. ${ }^{1}$ Hoffer et al randomized patients with venous outflow stenosis to either balloon angioplasty or Wallstent placement. While Wallstent placement increased the cost of the procedure by $90 \%$, no significant advantage to stent placement was observed after adequate dilatation by angioplasty. ${ }^{1}$ These results suggest that a period of clinical observation should follow adequate response to angioplasty. If the need for re-intervention should arise, graft dysfunction will usually be noted by attentive nephrologists during dialysis and communicated to the interventional radiology staff. In situations such as elastic recoil stenosis, early recurring stenosis, angioplasty-induced venous rupture, and kinked outflow veins, stent placement has been employed as a primary treatment.

Peripheral venous stents in hemodialysis patients tend to have similar primary and secondary patency rates, regardless of location (Table 1). ${ }^{2-6}$ Despite problems associated with compression and puncture of intra-shunt stents described later in the article, short-and mid-term patency rates are comparable to other locations of peripheral venous stent placement. Patency rates at the venous anastomosis tend to be lower than other sites. Funaki et al demonstrated that stents placed across femoral venous stenoses caused by arteriovenous grafts result in patency rates that are comparable or better than those achievable in upper extremity venous outflow stenoses. $^{2}$

Complications of peripheral venous stent placement in hemodialysis patients include misplacement, migration, stent fracture, and deformity. Zaleski et al described complications of mechanical compression and puncture-related deformity of intragraft stents caused by the peripheral location. Linear fractures of peripherally placed, self-expanding Wallstents were thought to be related to physical compression. ${ }^{4}$ Maleux et al described compression-induced collapse of a peripherally placed balloon-expandable Palmaz stent. ${ }^{5}$ Stents placed within dialysis shunts can undergo distortion and fracture from frequent puncture for dialysis. ${ }^{4}$ Finally, the most serious complication of peripheral stent placement is the migration of peripheral venous stents to the right atrium, right ventricle, and pulmonary arteries. ${ }^{6}$ The use of self-expanding stents and the practice of overestimating the diameter of the vein when choosing stent size may minimize the risk of this serious complication. ${ }^{7}$

\section{Treatment of Angioplasty-Induced Rupture}

Interventional radiologists should be familiar with those situations in which significant morbidity can result from delaying treatment with stents. Rupture of the venous outflow of arteriovenous shunts occurs in 2 to $3 \%$ of angioplasty procedures. ${ }^{8,9}$ The routine use of bare stents to salvage shunt thrombolysis complicated by venous rupture was first described by Funaki et al. ${ }^{9}$ Because the alternative for most patients would be loss of the dialysis shunt, stents are now indicated as a firstline treatment for venous rupture related to angioplasty. For this reason, when performing post-angioplasty venograms of dialysis grafts and fistulae, I recommend maintaining wire access across all stenotic lesions prone to rupture-especially tight lesions or lesions at the

Table 1 Treatment of Dialysis Graft Stenoses with Stents

\begin{tabular}{|c|c|c|c|c|c|c|}
\hline \multirow[b]{2}{*}{ Author } & \multirow[b]{2}{*}{ Year } & \multirow[b]{2}{*}{ No. of Grafts } & \multirow[b]{2}{*}{ Indication } & \multicolumn{3}{|c|}{ Primary/Secondary Assisted Patency Rates (\%) } \\
\hline & & & & $6 \mathrm{mo}$ & $12 \mathrm{mo}$ & $18 \mathrm{mo}$ \\
\hline Patel et $\mathrm{al}^{3}$ & 1999 & 25 & Venous anastomosis & $27 / 72$ & n.a./50 & n.a. \\
\hline Funaki et $\mathrm{al}^{2}$ & 1999 & 20 & Femoral venous outflow & $87 / 95$ & $51 / 92$ & $39 / 81$ \\
\hline Zaleski et al ${ }^{4}$ & 2001 & 11 & Intra-graft & $36 / 91$ & $12 / 71$ & $12 / 47$ \\
\hline Rajan et $\mathrm{al}^{5}$ & 2003 & 9 & Venous outflow rupture & $33 / 76$ & $17 / 69$ & n.a. \\
\hline Rajan et al ${ }^{6}$ & 2003 & 17 & Cephalic arch & 83/n.a. & 75/n.a. & n.a. \\
\hline
\end{tabular}

n.a., not available. 
cephalic arch ${ }^{10}$ Anticipating stent placement in these cases will prevent unnecessary morbidity caused by shunt loss or expanding hematoma.

While the mechanism for sealing perforations related to venous angioplasty using bare stents is unknown, Funaki et al postulated that the resistance to forward blood flow is less than the resistance to lateral extravasation. ${ }^{9}$ Therefore, the stent provides a lowpressure conduit through which blood can flow. Support for this mechanism comes from the fact that this treatment is successful despite the presence of therapeutic blood levels of anticoagulant and thrombolytic medications, preventing healing of the venous rift by the normal coagulation cascade. As Table 1 shows, patency rates following stent placement across venous ruptures are comparable to those following venous stenting for other indications. 8,9

\section{Treatment of Pseudoaneurysms}

Pseudoaneurysm formation is a common occurrence in patients with dialysis shunts, particularly native fistulae. They are usually caused by frequent punctures at the same location at the time of dialysis, ${ }^{11}$ despite the fact that Guidelines 27 and 28 of the Dialysis Outcomes Quality Initiative (DOQI. Available at: www.kidney.org/professionls/kdoqi) recommend avoiding needle insertion into pseudoaneurysms. In many cases, the dialysis access remains functional despite multiple, grossly enlarged pseudoaneurysms. Occasionally, saccular deformity of the venous outflow results in difficult shunt access during dialysis and even thrombosis due to the turbulence of flow. In addition, the presence of multiple large pseudoaneurysms can become a cosmetic problem for the patient.

In native dialysis fistulae, I do not recommend the use of covered stents as a cosmetic treatment for pseudoaneurysm formation. Functioning fistulae may remain patent for years, despite the presence of pseudoaneurysms, and placement of any stent will likely compromise long-term patency by inducing the formation of intimal hyperplasia. However, in cases of pseudoaneurysm formation leading to shunt thrombosis or difficulty accessing the shunt for dialysis, covered stents may be indicated. Any cosmetic benefit in these cases would be secondary to the principle role of the covered stent- to lengthen the patient's life by preserving functional access. DOQI Guideline 28 also recommends a conservative approach - revision of the fistula only if the pseudoaneurysm involves and compromises the arterial anastomosis. As covered stents become more widely used, DOQI recommendations may expand to include this relatively new technology.

In synthetic dialysis grafts, pseudoaneurysm formation is less common and less pronounced. DOQI Guideline 27 recommends resection and surgical inser- tion of an interposition graft if the pseudoaneurysm is infected, threatening the overlying skin, exceeds twice the diameter of the graft, or is rapidly expanding in size. Because the materials used in covered stents are similar or identical to the materials used for the synthetic grafts themselves, the use of covered stents for treating pseudoaneurysms is very promising. In addition, the application of covered stents is most likely less costly and less invasive than surgical revision.

Preliminary results published by Ryan at al in four cases suggested that Wallgrafts placed across pseudoaneurysms could be punctured without deformity or recurrence. ${ }^{12}$ However, as discussed previously, the distortion and fracture of bare stents placed within dialysis grafts has been described in the literature, ${ }^{4}$ and this phenomenon can compromise graft function. Further study is warranted to determine if these problems will occur with covered stents in the long term. Rabindranauth et al placed covered Palmaz balloon-expandable stents in 2 patients with polytetetrafluoroethylene (PTFE) bridge graft fistulae and reported continued patency in both after 6 months. ${ }^{13}$ Najibi et al placed Wallgrafts in 10 patients with either arteriovenous grafts or fistulae, and noted persistent patency in 7 patients at 6 months. ${ }^{14}$ Covered stents placed in hemodialysis shunts are discussed further later in the article.

\section{CENTRAL VENOUS STENOSIS}

Central venous stenosis related to hemodialysis access is a serious problem, not only threatening future dialysis access options, but also producing debilitating clinical symptoms. Patients present with swelling of the ipsilateral extremity, face, neck, and chest, as well as unsightly dilation of subcutaneous chest veins. In all cases, treatment should be initiated to minimize morbidity, improve the patient's immediate clinical symptoms, and to prolong life through preservation of hemodialysis access. Abandonment of dialysis access or compromise of future dialysis access sites should only be considered when no other therapeutic option exists.

Prior to the advent of minimally invasive treatments, surgery was the only option for central venous stenosis, but central venous bypass has become increasingly rare as a primary treatment. Because thoracotomy is required, central venous bypass and right atrial bypass grafting carry significant risk of morbidity and mortality, especially in chronically ill patients with end-stage renal disease. Surgery can be successful as an extreme measure in young or otherwise healthy patients, and is usually recommended for patients who have exhausted all access sites and minimally invasive options. ${ }^{15}$

In cases of central venous stenosis without complete occlusion, the primary treatment options include angioplasty and stent placement. As in cases of peripheral venous outflow stenosis, the most common approach 
to central stenosis is to employ angioplasty as a first-line treatment. Angioplasty is safe and inexpensive compared with the cost of stent placement ${ }^{4}$ and, in my experience, results in primary patency rates comparable to stent placement in carefully selected cases. If persistent improvement of the stenosis is achieved immediately following angioplasty, the case warrants a period of clinical observation. If clinical symptoms resolve and the dialysis access remains patent, a stent becomes unnecessary, thus lowering cost and potential morbidity. At the University of Chicago Hospitals, we deploy stents in patients with early recurrence of clinical symptoms or early loss of hemodialysis shunt patency following angioplastyusually within 4 weeks. Later recurrences can be treated safely and easily with repeat angioplasty procedures, as needed. For patients with complete central venous occlusion or elastic recoil of central venous stenosis immediately following angioplasty, we use stents as the primary treatment.

In 1997, Lumsden et al reported primary assisted patency rates of $42 \%$ at 6 months and $17 \%$ at 1 year in the central veins, ${ }^{16}$ and described these early results as "not optimistic." Over the past 5 years, primary and secondary assisted patency rates for central venous stenosis have become increasingly well defined, and marked improvement over the initial results has been achieved (Table 2). ${ }^{9,17-19}$ In addition, even temporary patency achieved through this minimally invasive treatment has significant implications for patients with chronic renal failure in terms of morbidity and mortality.

Complications of central venous stent placement have included compression or distortion of the stent at the thoracic inlet, central venous rupture with or without cardiac tamponade, adherence of tunneled catheters to the stent, ${ }^{20}$ misplacement, and migration. Though uncommon, serious complications associated with both balloon-expandable and self-expanding stents have been described. Most interventional radiologists use self-expanding stents in the central veins because of a perceived risk of migration of balloon-expandable stents to the right atrium - arguably the greatest risk to central venous stent placement. Because no large studies have accurately described the incidence of right atrial migration, this potentially catastrophic complication is likely under-reported in relation to hemodialysis patients. However, numerous case reports have described stent migration of both balloon-expandable and self-expanding stents in association with a variety of procedure types. Prahlow et al described cardiac perforation in minutes due to Wallstent embolization to the right atrium during transhepatic portosystemic shunt placement. ${ }^{21}$ Bartorelli et al describe Palmaz embolization after superior vena cava stenting, successfully treated by percutaneous capture and redeployment in the iliac vein. $^{22} \mathrm{~A}$ variety of techniques using balloons, snares and wires have been described for management of misplaced or migrated central venous stents, ${ }^{23}$ including repositioning and deploying in an alternate location, retrieval, and surgical removal. Besides migration, both central venous and arterial rupture has occurred during central vein stent placement. Like stent migration, these serious complications have been associated with both balloon-expandable and self-expanding stents. Evans et al described aortic laceration during deployment of a Palmaz stent to treat benign superior vena cava syndrome. ${ }^{24}$ Martin et al described fatal pericardial tamponade caused by perforation of Wallstent strut through the wall of the superior vena cava and into the pericardial space. ${ }^{25}$ Because of these scattered reports, stent preferences vary between physicians and institutions, but most investigators use self-expanding stents in the central venous circulation.

\section{ON THE HORIZON}

As the role of stents in the treatment of dialysis access complications has expanded, so has stent technology. While a discussion of the full range of stents available on the market is beyond the scope of this article, some new technologies are worth mentioning, including covered stents, brachytherapy, and drug-coated stents.

The application of covered stents to the treatment of pseudoaneurysms of vascular shunts was discussed previously. In addition, investigators have used covered stents for more traditional dialysis access applicationsincluding the treatment of venous outflow stenoses and occlusions. Preliminary results of covered stents in the peripheral venous circulation are limited. Farber et al achieved 12 -month primary and secondary patency rates of 29 and $64 \%,{ }^{26}$ suggesting that covered stents provide little advantage over bare stents for routine peripheral venous applications.

Table 2 Treatment of Central Venous Stenosis with Stents

\begin{tabular}{|c|c|c|c|c|c|}
\hline \multirow[b]{2}{*}{ Author } & \multirow[b]{2}{*}{ Year } & \multirow[b]{2}{*}{ No. of Grafts } & \multicolumn{3}{|c|}{ Primary/Secondary Assisted Patency Rates (\%) } \\
\hline & & & $3 \mathrm{mo}$ & $6 \mathrm{mo}$ & $12 \mathrm{mo}$ \\
\hline Vesely et $\mathrm{al}^{17}$ & 1997 & 20 & $88 / 89$ & $62 / 64$ & $47 / 56$ \\
\hline Haage et $\mathrm{al}^{9}$ & 2000 & 50 & 92/n.a. & $84 / 97$ & $56 / 97$ \\
\hline Verstandig et $\mathrm{al}^{18}$ & 2003 & 10 & n.a. & $66 / 100$ & $25 / 75$ \\
\hline Chen et $\mathrm{al}^{19}$ & 2003 & 18 & $100 / 100$ & 73/93 & $49 / 85$ \\
\hline
\end{tabular}

n.a., not available. 
Due to the similarity between tumor cell growth and benign tissue proliferation, new technologies for treating vascular restenosis focus on localized suppression of cell growth. While these technologies have been largely studied for use in intra-arterial applications, they promise tremendous applicability to stenoses of peripheral dialysis access. Initial results of stents coated with the immunosuppressive agent, sirolimus, show significant reductions in restenosis, ${ }^{27}$ and the coating of stents with other immunosuppressive drugs is on the immediate horizon. Brachytherapy, or local administration of intravascular radiation, has been successfully employed to suppress intimal hyperplasia after coronary angioplasty, and is more recently being applied to hemodialysis grafts. While case reports of brachytherapy applied to central venous stents in dialysis access patients have shown limited results, ${ }^{28}$ more extensive and systematic studies are underway. The ongoing BRAVO (Beta Radiation for Treatment of ArterialVenous Graft Outflow) trial is the first multi-center, randomized, double-blind, clinical trial of endovascular radiation in PTFE dialysis grafts, sponsored by the Novoste Corporation.

Preliminary work is underway at multiple institutions to design biodegradable stents with endovascular applications. Animal studies of expandable poly (L-lactic acid) stents have demonstrated the feasibility of design and intra-arterial deployment by conventional means, but a strong inflammatory response to this device is currently under study. ${ }^{29}$ In the future, biodegradable polymer technology may be combined with immunosuppressive drug-encoding ${ }^{30}$ to greatly enhance the maintenance of peripheral dialysis access.

\section{GENERAL GUIDELINES FOR STENT PLACEMENT IN HEMODIALYSIS PATIENTS}

In all patients confined to permanent hemodialysis, the treatment goal is the same-to lengthen the patient's lifespan to the greatest extent possible by preserving dialysis access options, maintaining existing dialysis access, and utilizing the most minimally invasive means available. To this end, investigators have offered suggestions to prevent loss of future access options, venous occlusion, stent migration, and infection.

\section{Bridging Side Veins}

Unless absolutely necessary to resolve severe clinical symptoms of venous obstruction, bridging side branches should be avoided to prevent loss of future dialysis access options.

Indiscriminate stent placement across peripheral venous outflow sites can obstruct future sites for arteriovenous shunt and graft placement. Common examples of this pitfall include routine stent placement across the venous anastomosis into the native vein, and routine stent placement from the cephalic arch into the subclavian vein. In both cases, alternate drainage pathways peripheral to the stent are compromised. In the case of central venous occlusion, unnecessary coverage of jugular or brachiocephalic vein inflow can have dire consequences, limiting the patient's lifespan by compromising numerous catheter and shunt options.

\section{Venous Occlusion}

While I do not routinely use anticoagulation unless the patient is undergoing concurrent dialysis shunt thrombolysis, some radiologists have prescribed the routine short-term use of anticoagulation to minimize the risk of early thrombosis following stent placement. We have found that in most patients with dialysis shunts, flow rates are adequate to maintain patency following stent placement. We usually reserve anticoagulation for those patients with long, irregular lesions, or a suboptimal result following stent placement.

To further reduce the risk of early thrombosis following stent placement, most investigators have advocated the use of the shortest stent possible to entirely cover the stenosis. Because the stent can be a nidus for early thrombosis as well as for later formation of intimal hyperplasia, the use of a shorter stent may avoid both early and delayed complications of outflow occlusion.

\section{Stent Migration}

In the case of central venous stent placement, stent migration has been associated with significant morbidity, and even mortality. We have found that superior vena cava Wallstent placement from the femoral approach, or inferior vena cava Wallstent placement from the arm approach maximizes leverage and minimizes the risk of stent migration. In each case, migration of the stent toward the right atrium during deployment is rare and easily correctible by stent recapture.

Generally, most investigators recommend selfexpanding stents for central venous stenosis, most commonly the Wallstent. These stents come in large sizes for optimal overestimation of the normal lumen size. In addition, self-expanding stents often continue to enlarge after deployment in patients with elastic recoil stenosis. In our opinion, the use of balloon-expandable stents for central venous stenosis poses an unnecessary risk of catastrophic stent migration to the right atrium, especially if the normal vessel lumen size is miscalculated or the stent is incompletely expanded due to balloon rupture. For similar reasons, we have used self-expanding stents in the peripheral veins with the occasional exception of stenosis at the venous anastomosis of arteriovenous grafts. At this location, balloon-expandable stents offer the occasional advantage of providing 
extremely focal coverage, avoiding unnecessary coverage of the native outflow vein.

\section{Infection}

While the infection rate after stent deployment is extremely low, ${ }^{31}$ it is prudent to delay stent placement in all patients that are potentially bacteremic. We routinely cover patients with antibiotics to avoid graft infection during revision or thrombolysis. However, some investigators have reported little or no incidence of infection related to stent placement, despite the lack of routine antibiotic use.

\section{REFERENCES}

1. Hoffer EK, Sultan S, Herskowitz MM, et al. Prospective randomized trial of a metallic intravascular stent in hemodialysis graft maintenance. J Vasc Interv Radiol 1997;8:965-973

2. Funaki B, Szymski GX, Leef JA, et al. Treatment of venous outflow stenoses in thigh grafts with Wallstents. AJR Am J Roentgenol 1999;172:1591-1596

3. Patel RI, Peck SH, Cooper SG, et al. Patency of Wallstents placed across the venous anastomosis of hemodialysis grafts after percutaneous recanalization. Radiology 1998;209:365370

4. Zaleski GX, Funaki B, Rosenblum J, et al. Metallic stents deployed in synthetic arteriovenous hemodialysis grafts. AJR Am J Roentgenol 2001;176:1515-1519

5. Maleux G, Rousseau H, Otal P, et al. Collapsed Palmaz stent after deployment for hemodialysis access-related venous stenosis. J Vasc Interv Radiol 1998;9(1 Pt 1):169-171

6. Asher RM, Huettl EA, Halligan R. Percutaneous retrieval of a Wallstent from the pulmonary artery following stent migration from the iliac vein. J Interv Cardiol 2002;15(2): 101-106

7. Haage P, Vorwerk D, Piroth W, Schuermann K, Guenther RW. Treatment of hemodialysis-related central venous stenosis or occlusion; results of primary Wallstent placement and follow-up in 50 patients. Radiology 1999;212:175-180

8. Rajan DK, Clark TW. Patency of Wallstents placed at the venous anastomosis of dialysis grafts for salvage of angioplastyinduced rupture. Cardiovasc Intervent Radiol 2003;26: 242-245

9. Funaki B, Szymski GX, Leef JA, Rosenblum JD, Burke R, Hackworth CA. Wallstent deployment to salvage dialysis graft thrombolysis complicated by venous rupture: early and intermediate results. AJR Am J Roentgenol 1997;169: $1435-1437$

10. Rajan DK, Clark TW, Patel NK, et al. Prevalence and treatment of cephalic arch stenosis in dysfunctional autologous hemodialysis fistulas. J Vasc Interv Radiol 2003;14(5):567-573

11. Delorme JM, Guidoin R, Canizales S, et al. Vascular access for hemodialysis: pathologic features of surgically excised PTFE grafts. Ann Vasc Surg 1992;6:517-524

12. Ryan JM, Siobhan DA, Doherty J, et al. Using a covered stent (Wallgraft) to treat pseudoaneurysms of dialysis grafts and fistulas. AJR Am J Roentgenol 2003;180:1067-1071

13. Rabindranauth $P$, Shindelman L. Transluminal stent-graft repair for pseudoaneurysm of PTFE hemodialysis grafts. J Endovasc Surg 1998;5(2):138-141
14. Najibi S, Bush RL, Terramani TT, et al. Covered stent exclusion of dialysis access pseudoaneurysms. J Surg Res 2002; 106(1):15-19

15. El-Sabrout RA, Duncan JM. Right atrial bypass grafting for central venous obstruction associated with dialysis access: another treatment option. J Vasc Surg 1999;30(3):582-584

16. Lumsden $\mathrm{AB}, \mathrm{MacDonald} \mathrm{MJ}$, Isiklar $\mathrm{H}$, et al. Central venous stenosis in the hemodialysis patient: incidence and efficacy of endovascular treatment. Cardiovasc Surg 1997; 5(5):504-509

17. Vesely TM, Hovespian DM, Pilgram TK, et al. Upper extremity central venous obstruction in hemodialysis patients: treatment with Wallstents. Radiology 1997;204(2):343-348

18. Verstandig AG, Bloom AI, Sasson T, et al. Shortening and migration of Wallstents after stenting of central venous stenoses in hemodialysis patients. Cardiovasc Intervent Radiol 2003;26(1):58-64

19. Chen CY, Liang HL, Pan HB, et al. Metallic stenting for treatment of central venous obstruction in hemodialysis patients. J Chin Med Assoc 2003;66(3):166-172

20. McIntyre CW, Taal MW, Fluck RJ, et al. Adherence of tunneled haemodialysis catheter to superior vena caval stent: successful percutaneous removal. Nephrol Dial Transplant 2003;18:432-433

21. Prahlow JA, O'Bryant TJ, Barnard JJ. Cardiac perforation due to Wallstent embolization: a fatal complication of the transjugular intrahepatic portosystemic shunt procedure. Radiology 1997;205(1):170-172

22. Bartorelli AL, Fabbiocchi F, Montorsi P, et al. Successful transcatheter management of Palmaz stent embolization after superior vena cava stenting. Cathet Cardiovasc Diagn 1995; 34(2):162-166

23. Slonim SM, Dake MD, Razavi MK, et al. Management of misplaced or migrated endovascular stents. J Vasc Interv Radiol 1999;10(7):851-859

24. Evans J, Saba Z, Rosenfeld H, et al. Aortic laceration secondary to Palmaz stent placement for treatment of superior vena cava syndrome. Catheter Cardiovasc Interv 2000;49(2): 160-162

25. Martin M, Baumgartner I, Kolb M, et al. Fatal pericardial tamponade after Wallstent implantation for malignant superior vena cava syndrome. J Endovasc Ther 2002;9(5): 680-684

26. Farber A, Barbey MM, Grunert JH, et al. Access-related venous stenoses and occlusions: treatment with percutaneous transluminal angioplasty and Dacron-covered stents. Cardiovasc Intervent Radiol 1999;22(3):214-218

27. Morice MC, Serruys PW, Sousa JE, et al. A randomized comparison of a sirolimus-eluting stent with a standard stent for coronary revascularization. N Engl J Med 2002;346:17731780

28. Kwok PC, Wong KM, Ngan RK, et al. Prevention of recurrent central venous stenosis using endovascular irradiation following stent placement in hemodialysis patients. Cardiovasc Intervent Radiol 2001;24(6):400-406

29. Su SH, Chao RY, Landau CL, et al. Expandable bioresorbable endovascular stent. Fabrication and properties. Ann Biomed Eng 2003;31(6):667-677

30. Fattori R, Piva T. Drug-eluting stents in vascular intervention. Lancet 2003;361(9353):247-249

31. Guest SS, Kirsch CM, Baxter R, et al. Infection of a subclavian venous stent in a hemodialysis patient. Am J Kidney Dis 1995;26(2):377-380 